\title{
EFECTO DE LA COBERTURA DE RASTROJOS EN LA GERMINACIÓN DEL ARROZ (Oryza sativa L.) Y PRINCIPALES MALEZAS ASOCIADAS1
}

\author{
Yemel M. Ortega ${ }^{2}$, Renán Agüero Alvarado 3
}

\begin{abstract}
RESUMEN
Efecto de la cobertura de rastrojos en la germinación del arroz (Oryza sativa L.) y principales malezas asociadas. Para determinar el grado de interferencia causado por distintas cantidades de rastrojos de arroz (Oryza sativa L.) sobre la emergencia de arroz y malezas asociadas al cultivo, en el 2003 se realizaron tres experimentos simultáneos en la hacienda el Pelón de la Bajura, Guanacaste, Costa Rica. Se aplicaron nueve tratamientos ( 0 a $9 \mathrm{t} / \mathrm{ha}$ ) de coberturas con rastrojos de la variedad de arroz CR 1113 para medir los efectos en la emergencia del arroz y malezas. Las malezas asociadas incluyen las más frecuentes en agroecosistemas arroceros inundados: Oryza sativa, Echinochloa colona, Cyperus spp., Fimbristylis spp., Ludwigia spp. y Heteranthera limosa. En los primeros dos experimentos se determinaron relaciones inversas entre el grado de cobertura y la densidad de plántulas de malezas emergidas $\left(R^{2}>0,89\right)$, y entre el grado de cobertura y el porcentaje de germinación del arroz $\left(\mathrm{R}^{2}=0,86\right)$, respectivamente. Usando coberturas con rastrojos de frijol (Phaseolus vulgaris) en el tercer experimento, no se observó efecto supresor en la germinación del arroz, probablemente debido a su pronta descomposición. Aunque la germinación del arroz no se afectó aún a las intensidades más altas de rastrojos evaluadas, conviene realizar pruebas similares a largo plazo para determinar posibles efectos debidos a la descomposición de residuos, alelopatía y acarreo de herbicidas después de varios ciclos consecutivos del cultivo de arroz.
\end{abstract}

Palabras claves: Arroz (Oryza sativa L.), arroz rojo, rastrojos, emergencia, malezas.

\begin{abstract}
Crop residues effect on the germination of rice (Oryza sativa L.) and its major weeds. Three simultaneous experiments were conducted in El Pelón de la Bajura Farm, Guanacaste, Costa Rica in 2003 to determine the degree of interference caused by varying amounts of previous rice crop (Oryza sativa L.) residue on the emergence of rice and associated weeds. Nine coverage treatments of CR1113 crop residues ( 0 to $9 \mathrm{t} / \mathrm{ha}$ ) were applied to measure effects on rice and weed germination. The associated weeds included several of the most frequent in flooded rice: Oryza sativa, Echinochloa colona, Cyperus spp., Fimbristylis spp., Ludwigia spp. and Heteranthera limosa. In the first two experiments, inverse relationships were established between the degree of coverage and the density of emergent weed seedlings $\left(\mathrm{R}^{2}>0.89\right)$ and the degree of coverage and the percentage of rice germination $\left(\mathrm{R}^{2}=0.86\right)$, respectively. Using bean residue (Phaseolus vulgaris) in the third experiment but no suppressing effect on the germination of rice was observed, possibly due to its rapid decomposition. Although the germination of rice was not affected even at the highest level of residues, similar long-term trials should be conducted to evaluate the possible impact of residue decomposition, allelopathy and herbicide carry-over effects after various consecutive rice production cycles.
\end{abstract}

Key words: Rice (Oryza sativa L.), red rice, stubble, emergence, weeds.

\section{INTRODUCCIÓN}

Tras la evolución de la agricultura durante las últimas cinco décadas y la generación de conocimientos sobre distintas formas de cultivo, se pueden esbozar dos posiciones extremas; una donde la producción agrícola está fundamentada en la preparación física del terreno hasta dejarlo finamente pulverizado, en la cual el manejo del cultivo se basa en aplicaciones de fertilizantes y pesticidas químicos (Temple et al. 1994), y la otra donde

\footnotetext{
1 Recibido: el 11 de noviembre, 2004. Aceptado: 28 de marzo, 2005. Parte de la Tesis de doctorado del primer autor, en Sistemas de Producción Agrícola Tropical Sostenible, Universidad de Costa Rica.

2 Programa de Doctorado en Sistemas de Producción Agrícola Tropical Sostenible, Universidad de Costa Rica; yemel.ortega@edifystudios.com

3 Facultad de Ciencias Agroalimentarias, Universidad de Costa Rica; raguero@cariari.ucr.ac.cr
} 
se cultiva sin necesidad de alterar el suelo y prácticamente sin efectuar aplicaciones de agroquímicos, permitiendo que las plantas crezcan con la mínima intervención humana (Fukuoka 1978).

En medio de estas posiciones extremas es posible diferenciar una gran cantidad de sistemas alternativos de producción agrícola, que combinan diferentes niveles de preparación física del terreno y aplicación de agroquímicos. Dentro de estos sistemas se encuentran la labranza mecanizada convencional (LMC) y la labranza cero, también conocida como siembra directa sobre rastrojos (SDR). El grado de intervención al suelo, así como la cantidad y cobertura de rastrojos remanentes sobre el suelo son los factores de mayor relevancia en la definición de estos sistemas.

Los rastrojos son plantas controladas y material vegetal que queda en el terreno del ciclo anterior, una vez realizada la cosecha del cultivo principal. Éstos incluyen tanto la parte aérea (principalmente tallos y hojas) como radicular de la soca ${ }^{1}$ y de las malezas presentes en el agroecosistema cuyo crecimiento ha sido suprimido, así como residuos vegetales remanentes en forma separada sobre el suelo (caña y paja de arroz). El porcentaje de cobertura de rastrojos se emplea como criterio para clasificar los sistemas de cultivo arriba citados por ser un factor importante en el control de la erosión del suelo.

En la siembra mecanizada convencional los rastrojos son eliminados por completo a través de su incorporación al perfil del suelo durante las operaciones de labranza (Jasa et al. 2000). La degradación anaeróbica de los rastrojos incorporados en campos arroceros inundados libera alrededor de 60 a 100 millones de t de metano $\left(\mathrm{CH}_{4}\right)$, contribuyendo substancialmente al calentamiento global de la atmósfera (Weber et al. 2001).

La labranza reducida se refiere a cualquier sistema que sea menos intensivo y agresivo que la labranza convencional, con menor cantidad de operaciones y consumo de energía por unidad de área. La efectividad de este sistema para conservar el suelo depende principalmente de la proporción de terreno cubierto con rastrojos. En este sentido, una cobertura de 20 a $30 \%$ reduce la erosión en $50 \%$ mientras que si la misma es del $70 \%$, la erosión puede reducirse en más del $90 \%$ en comparación con un suelo sin cobertura (Simmons 2002).

\footnotetext{
1 La soca se define como plantas cosechadas con potencial para iniciar un nuevo ciclo productivo y generar una nueva cosecha. La soca es común en cultivos perennes como la caña de azúcar, así como en algunos biotipos de cultivos anuales como en el arroz.
}

En algunos sistemas de labranza reducida como el arado de cincel, labranza con disco rotatorio y la labranza en contorno es preciso mantener al menos $30 \%$ de cobertura con residuos de cosecha para reducir la erosión después del cultivo de granos como maíz, sorgo y arroz (Smith et al. 2000). La siembra directa sobre rastrojos $^{2}$ es un tipo particular de labranza reducida en el cual el suelo es únicamente disturbado por la sembradora al momento de cortar los rastrojos y abrir pequeños surcos en el suelo para depositar la semilla del cultivo o incorporar fertilizantes. En este sistema es esencial conservar y manejar en forma apropiada prácticamente todos los residuos remanentes del ciclo previo.

Esta cobertura vegetal protege la superficie del suelo de la erosión absorbiendo la energía de impacto de las gotas de lluvia y así se reduce el desprendimiento de partículas y la formación de superficies endurecidas. Al obstruir los flujos superficiales, los rastrojos reducen la velocidad de la escorrentía y la cantidad de suelo transportado, a la vez que minimizan los desprendimientos adicionales de partículas y mejoran la infiltración del agua. Cuando la velocidad del flujo es disminuida, muchas de las partículas de suelo en suspensión quedan depositadas nuevamente sobre el terreno. Adicionalmente los rastrojos mantienen atrapada bajo su cobertura la humedad, propiciando mejores condiciones para la germinación del cultivo en años de lluvias escasas. También protegen al suelo y las plántulas del sol a medida que el nuevo cultivo crece, reduciendo las pérdidas de agua por evaporación y transpiración (Bull 1993; Shelton et al. 2000a; Smith et al. 2000).

La cantidad y distribución de rastrojos después de la siembra depende del tipo de cultivo anterior y los implementos utilizados para la cosecha. Después de una cosecha de arroz son típicas coberturas del orden de 60 a 70\% (Bull 1993). En general, mientras más altos sean los rendimientos del cultivo mayor será la cantidad de material vegetal producido. En arroz esta cantidad es directamente proporcional al rendimiento en granza. Por cada tonelada de grano cosechada se produce una cantidad equivalente de residuos (Cordero 1993), con proporciones entre 0,8 a 1 y 1,2 a 1 , lo cual puede variar de 2 a más de 8 t/ha dependiendo de la variedad, productividad y método de la cosecha.

La distribución uniforme del material cortado por la cosechadora combinada es un factor esencial para lograr una cobertura homogénea del suelo y evitar deficiencias en el control de malezas. Cuando la distribución de los

\footnotetext{
2 Cero labranza o siembra directa sobre rastrojos es empleado en este estudio como sinónimo del concepto "No-Till”, en el cual la labranza es eliminada o aplicada al mínimo posible.
} 
rastrojos es dispareja se torna más difícil cortarlos y depositar la semilla del cultivo sin ocasionar disturbios significativos al suelo (Smith et al. 2000). Si la sembradora no puede cortar o separar capas gruesas, es posible que se entierren tallos del cultivo anterior junto con las semillas. En estos casos la semilla del cultivo no logra buen contacto con el suelo, reduciendo la germinación y provocando hileras discontinuas.

Al igual que en caña de azúcar, sorgo y maíz, los rastrojos del arroz se clasifican como no frágiles. Expuestos a la intemperie estos rastrojos resisten más la descomposición que los denominados frágiles (frijol, maní, etc.). En el caso particular del arroz, el rastrojo está formado en gran parte por tallos fibrosos y muy resistentes. Debido a estas características, en muchas regiones del mundo los tallos de arroz son usados para elaborar alfombras, mecates y zapatos. Adicionalmente, por tener alta resistencia a la descomposición bacteriana sirven también como material de construcción (Yang y Kim 2003).

Los rastrojos de arroz son difíciles de descomponer porque tienen elevados contenidos de sílice que pueden oscilar de 8,8 a 13,3\% según la variedad (Fadel y MacKill 1994; Quick 2003). Además del sílice, los principales componentes de estos son hemicelulosa, celulosa y lignina. Debido a que poseen un contenido de cenizas superior al $22 \%$ del peso total, estos no se descomponen tan rápidamente como los de otros granos básicos. Al momento de la cosecha su contenido de humedad usualmente es superior al $60 \%$, pero cuando el tiempo es seco rápidamente baja hasta un punto de equilibrio entre 10 y $12 \%$. Con características como las apuntadas, al adoptar el sistema SDR es preciso definir manejos particulares para este tipo de residuo, y entender su efecto sobre la emergencia del arroz, arroz rojo y otras malezas asociadas al cultivo.

Los beneficios derivados por la presencia de rastrojos sobre el suelo varían según sea la durabilidad de los materiales vegetales que los constituyen. La velocidad de descomposición de los rastrojos es afectada por el tamaño y la distribución de éstos (Johnston y Miller 1973). Cuando los tallos del arroz son cortados en partes pequeñas y distribuidos uniformemente su descomposición ocurre con mayor rapidez, ya que los microorganismos del suelo degradan y reducen la superficie cubierta con éstos, especialmente hojas y pedazos pequeños. La humedad y altas temperaturas incrementan la tasa a la cual ocurre esa degradación, lo cual es común en ambientes inundados con alternancia de períodos húmedos y secos.

Distinto a lo que ocurre con estructuras frágiles de plantas leguminosas como el frijol, en residuos vegeta- les de arroz estos procesos se desarrollan con menor rapidez y la cobertura protectora sobre el suelo permanece por más tiempo. Sin embargo, cuando los tallos de arroz son picados en pedazos pequeños y redistribuidos por difusores acoplados a combinadas, los rastrojos son menos resistentes a la descomposición (Shelton et al. 2000b).

Debido a que el uso de coberturas puede reducir el uso de herbicidas y la movilidad de agroquímicos aplicados al cultivo, se consideran como un componente importante en el desarrollo de sistemas agrícolas sostenibles. La supresión de malezas por esta vía es atribuida a interferencias físicas y químicas. Dentro de las interferencias físicas se destaca la luz como un factor que estimula la germinación de muchas semillas de malezas (Penny y Neal 2003). En SDR al quedar el suelo cubierto se reduce la emergencia de malezas que requieren luz para su germinación. Otros factores importantes para activar la germinación de las semillas y emergencia de plántulas son la temperatura y la humedad.

La capa vegetal afecta la germinación, emergencia y dinámica de malezas sensibles a la cobertura del suelo. Cuando el terreno está cubierto se altera la luminosidad, temperatura y humedad del suelo, interfiriendo con la dormancia de semillas de especies sensibles a estos factores. Adicionalmente se puede afectar el desarrollo de malezas y del cultivo al constituir una barrera física que induce etiolación y daños mecánicos durante la emergencia de las plántulas (Correia y Durigan 2004). Aunque los rastrojos no proporcionan control total de malezas (Teasdale 1996), sí pueden reducir el uso de herbicidas pre y posemergentes, permitiendo mejorar los programas de control de plantas indeseables. En consecuencia, el manejo de coberturas es un aspecto importante para el desarrollo de programas de control integrado de plagas.

Si bien la cobertura proporciona importantes beneficios al agroecosistema, existe evidencia de que los residuos del arroz algunas veces pueden inhibir el crecimiento de malezas y cultivos subsecuentes, incluyendo al mismo arroz. En este último caso, en el proceso llamado autointoxicación, las fitotoxinas producidas por los restos vegetales del arroz pueden limitar el crecimiento y el tamaño de la población del propio cultivo. En Taiwán un 25\% de la reducción en el rendimiento de arroz en un segundo ciclo fue atribuida principalmente a la producción de fitotoxinas producidas por la descomposición de los residuos dejados en el suelo. En Filipinas hay preocupación por impactos a largo plazo, pues un estancamiento en la productividad del arroz ha sido atribuido a cambios en la disponibilidad de nitrógeno, causada por la cantidad masiva de desechos acumulados y sin incorporar al suelo, luego de ciclos consecutivos y la inundación continua (Chow 1998). 
El principal objetivo del presente estudio fue evaluar el efecto de diversos niveles de cobertura con rastrojos de arroz sobre la germinación tanto de las malezas más comunes de un agroecosistema arrocero inundado, como del arroz. Cuando se aplica en forma continua el sistema de SDR es importante conocer estos efectos para efectuar los ajustes pertinentes en la cantidad de residuos de cosecha que quedan en el campo y la cantidad de semillas a usar durante la siembra del arroz. De esta forma es posible adecuar el control de malezas, complementando diferentes estrategias de control.

\section{MATERIALES Y MÉTODOS}

\section{El sitio del estudio}

La fase experimental de esta investigación se realizó entre julio y noviembre del 2003 en una casa de mallas de la hacienda el Pelón de la Bajura, en Guanacaste, Costa Rica. El sitio del estudio está ubicado entre las coordenadas verticales 384-385 y horizontales 265-266 de la hoja cartográfica Tempisque, a $40 \mathrm{msnm}$ (IGN 1987), en la zona de vida bosque húmedo premontano (CCT 1993). Según datos de la estación hidrometeorológica instalada en la misma hacienda, la temperatura media anual fue de $27,6{ }^{\circ} \mathrm{C}$, con promedios mínimos y máximos respectivos de 22,9 y $34,7^{\circ} \mathrm{C}$. La humedad relativa varió entre 61 y $90 \%$. Además del riego artificial la precipitación media en la finca (1722 mm/año), con el $66 \%$ de las lluvias concentradas en junio, setiembre y octubre, permitió mantener la inundación típica utilizada para la producción del cultivo a escala comercial.

\section{Diseño experimental}

El estudio consistió en tres experimentos complementarios que se llevaron a cabo en forma simultánea para determinar el efecto de distintas cantidades de dos tipos de coberturas en la emergencia del arroz y malezas importantes asociadas al cultivo. En los dos primeros experimentos se evaluó la emergencia de las malezas más comunes de agroecosistemas arroceros de la región y la emergencia de plántulas de arroz, respectivamente. En ambos casos, se utilizaron diferentes niveles de rastrojos de arroz como cobertura del suelo.

En el tercer experimento se evaluó el efecto de diferentes cantidades de coberturas con plantas de frijol en la emergencia del arroz. Si bien estos rastrojos son frágiles, se incluyeron en el estudio por la capacidad del frijol para fijar nitrógeno atmosférico que subsecuentemente quedaría disponible para el cultivo siguiente durante la descomposición de los residuos, y los benefi- cios de la rotación del cultivo de arroz con leguminosas (Reddy 2001).

En los tres experimentos se utilizó un diseño de bloques completos al azar, con nueve tratamientos y cuatro repeticiones. Las unidades experimentales fueron macetas plásticas con capacidad para albergar 500 cc de suelo. Se emplearon cinco macetas por tratamiento, rellenas con suelo recolectado del lote Playitas, en la capa del perfil del suelo comprendida entre 0 y 5 $\mathrm{cm}$ de profundidad. Los tratamientos consistieron en densidades variables de rastrojos cubriendo la superficie de las macetas, en el ámbito equivalente entre 0 y 9 t/ha, tanto para residuos no frágiles de arroz como en el caso de frágiles de frijol. Al definir dicho ámbito, se tomó como referencia la cantidad normal de rastrojos remanentes en el terreno, en condición seca luego de la cosecha del arroz CR1113, estimada en 4,5 t/ha en el presente estudio.

Para los experimentos uno y dos, se utilizó paja de arroz obtenida de plantas en pie de la variedad CR 1113 correspondientes a la cosechada de la siembra de verano 2002-2003. La paja fue puesta a secar bajo la exposición directa a la radiación solar, hasta lograr aproximadamente un contenido de humedad del $12 \%$, similar al alcanzado tras la cosecha en condiciones de campo. En el experimento 3, la cobertura consistió en follaje seco de frijol Negro Huasteco. Ambos fueron picados en tamaños inferiores a $1 \mathrm{~cm}^{2}$ y se determinaron los pesos equivalentes a cada tratamiento. En el experimento uno se aprovechó el banco de semillas presente en el suelo depositado en las macetas. Para lograr mayor uniformidad el suelo fue secado al sol, tamizado en una malla con 5 $\mathrm{mm}$ de luz y mezclado dentro de un barril plástico, antes de llenar cada maceta. Para evitar la germinación de malezas en los experimentos dos y tres se efectuó una aplicación preemergente con butaclor, antes de la siembra, con el equivalente a $2,4 \mathrm{~kg} / \mathrm{ha}$ de i.a. de la formulación $60 \mathrm{EC}$ de producto comercial (4 1/ha). En las pruebas de germinación de estos dos ensayos se usó semilla certificada de arroz variedad CR 1113, colocando 100 semillas viables en cada maceta, luego de un tratamiento pregerminativo mediante inmersión en agua durante 12 horas.

\section{Análisis estadístico}

A los 30, 60 y 90 días después de establecido el ensayo uno, se efectuó un recuento de plántulas de malezas emergidas, las cuales fueron registradas por especie en términos de plantas por $\mathrm{m}^{2}$ antes del correspondiente análisis de varianza. En los ensayos dos y tres, se realizó un solo recuento de plántulas de arroz variedad CR 1113 emergidas a los 30 días y se determinaron los porcentajes de germinación respectivos. Previo al análisis 
de varianza, los datos fueron transformados mediante la función arco seno de la raíz cuadrada, y se efectuó el análisis estadístico de las variables transformadas.

\section{RESULTADOS Y DISCUSIÓN}

\section{Emergencia de malezas bajo cobertura de rastrojos de arroz}

Los resultados del primer experimento mostraron que distintas cantidades de cobertura con rastrojos de arroz afectaron de manera significativa $(\mathrm{p} \leq 0,05)$ la germinación y emergencia de las malezas más comunes del agroecosistema arrocero. El Cuadro 1 presenta los datos consolidados de tres evaluaciones mensuales, correspondientes al total de plántulas emergidas de las seis malezas observadas con mayor frecuencia en las macetas. Las malezas más comunes mostraron una relación inversa entre la cantidad de semillas germinadas o plántulas emergidas (plantas $/ \mathrm{m}^{2}$ ) y la cantidad de cobertura con rastrojos de arroz (Cuadro 2). Esta cobertura suprimió la germinación de las principales malezas, especialmente cuando fue mayor a la cantidad equivalente al total producido por la variedad considerada al término de un ciclo de cultivo (4,5 t/ha). La lenta descomposición de estos materiales vegetales permitió que con coberturas superiores a la mencionada, el suelo contenido en las macetas permaneciera tapado con rastrojos durante todo el período en evaluación (90 dds), obstaculizando los procesos de germinación y emergencia de las semillas de malezas presentes.

Altos niveles de cobertura con rastrojos en SDR pueden limitar la germinación y retardar la emergencia de plántulas germinadas de distintas malezas (Besnier
1989). La barrera física formada por los rastrojos distribuidos sobre el suelo limita la luz solar que penetra en éste, amortiguando a la vez las fluctuaciones de temperatura y humedad en las capas del perfil más cercanas a la superficie. De esta manera, la cobertura de rastrojos puede influir en los procesos que afectan la germinación y emergencia del banco de semillas de malezas del suelo. En este experimento, el efecto supresor en la germinación de algunas malezas ocasionado por los rastrojos de arroz, pudo deberse en parte al efecto combinado resultante por la presencia de dicha barrera.

Las semillas sensibles a la luz en muchas especies de malezas requieren que sus fitocromos sean expuestos a períodos cortos e intermitentes de radiación solar para desactivar su dormancia y germinar (Shrestha et al. 2002). El fitocromo constituye un mecanismo a través del cual las semillas pueden responder en forma positiva a la calidad de la luz y negativamente ante la luz filtrada por los rastrojos. En su forma activa el fitocromo absorbe luz roja y roja lejana en la forma inactiva, pero este mecanismo puede ser reversible. Las semillas sensibles a la luz normalmente germinarán en terrenos descubiertos que reciben alta proporción de luz roja, pero permanecerán latentes bajo la sombra de los rastrojos (Salisbury y Ross 1992). Cuando un terreno es tratado mediante SDR, la cobertura de rastrojos filtra la luz y la relación entre luz roja/roja lejana cae hasta valores menores de 0,1 en la superficie del suelo. La luz que penetra en un suelo bajo rastrojos es fundamentalmente roja lejana, la cual puede promover estados de latencia en las semillas de muchas malezas, impidiendo su germinación.

En general, los rastrojos inhiben la emergencia de la mayoría de las malezas cuando se aplican en suficiente cantidad (Penfold 2003). En el caso de Oryza

Cuadro 1. Cantidad media de plántulas de malezas emergidas en potes, según tipo de maleza, por tratamiento de cobertura con rastrojos de arroz. Hacienda El Pelón, Guanacaste, Costa Rica. 2003.

\begin{tabular}{|c|c|c|c|c|c|c|c|c|}
\hline \multirow[t]{2}{*}{$\begin{array}{c}\text { Tratamiento } \\
\text { (T) }\end{array}$} & $\begin{array}{c}\text { Proporción respecto } \\
\text { al total } \mathbf{R}\end{array}$ & $\begin{array}{c}\text { Cobertura } \\
\text { t/ha }\end{array}$ & $\begin{array}{l}\text { Oryza sativa } \\
\quad \text { (rojo) }\end{array}$ & $\begin{array}{c}\text { Echinochloa } \\
\text { colona }\end{array}$ & $\begin{array}{c}\text { Cyperus } \\
\text { spp }\end{array}$ & $\begin{array}{c}\text { Fimbristylis } \\
\text { spp }\end{array}$ & Ludwigia & $\begin{array}{c}\text { Heteranthera } \\
\text { limosa }\end{array}$ \\
\hline & & & \multicolumn{6}{|c|}{ Plántulas emergidas / m² } \\
\hline 1 & 0 & 0 & 65 & 50 & 75 & 44 & 9 & 27 \\
\hline 2 & 0,25 & 1,1 & 65 & 46 & 73 & 42 & 9 & 26 \\
\hline 3 & 0,50 & 2,3 & 62 & 41 & 70 & 37 & 6 & 24 \\
\hline 4 & 0,75 & 3,4 & 45 & 34 & 65 & 28 & 3 & 17 \\
\hline 5 & 1,00 & 4,5 & 30 & 27 & 54 & 20 & 2 & 9 \\
\hline 6 & 1,25 & 5,6 & 21 & 18 & 42 & 13 & 2 & 7 \\
\hline 7 & 1,50 & 6,8 & 14 & 13 & 31 & 7 & 0 & 3 \\
\hline 8 & 1,75 & 7,9 & 11 & 10 & 23 & 4 & 0 & 0 \\
\hline 9 & 2,00 & 9,0 & 10 & 8 & 19 & 0 & 0 & 0 \\
\hline
\end{tabular}

Nota $\mathrm{R}=$ Cantidad de rastrojos remanentes en campo, estimada en 4,5 t/ha para arroz CR1113 en este estudio. 
Cuadro 2. Relación entre la cantidad de plántulas de malezas emergidas en potes (Y), según tipo de maleza, por tratamiento de cobertura con rastrojos de arroz (X). Hacienda. El Pelón, Guanacaste, Costa Rica. 2003.

\begin{tabular}{lllcc}
\hline Especie de maleza & \multicolumn{1}{c}{ Familia } & $\begin{array}{c}\text { Ecuación de } \\
\text { regresión lineal }\end{array}$ & $\begin{array}{c}\text { Coeficiente de } \\
\text { determinación }\left(\mathbf{R}^{2)}\right.\end{array}$ & $\begin{array}{c}\text { Nivel de } \\
\text { Significancia }\end{array}$ \\
\hline Oryza sativa & Poaceae & $\mathrm{Y}=69,37-7,42 \mathrm{X}$ & 0,94 & $* * *$ \\
Echinochloa colona & Poaceae & $\mathrm{Y}=50,66-5,14 \mathrm{X}$ & 0,97 & $* * *$ \\
Cyperus spp. & Cyperaceae & $\mathrm{Y}=81,91-7,02 \mathrm{X}$ & 0,96 & $* * *$ \\
Fimbristylis spp. & Cyperaceae & $\mathrm{Y}=46,02-5,40 \mathrm{X}$ & 0,98 & $* * *$ \\
Ludwigia spp. & Onagraceae & $\mathrm{Y}=8,52-1,12 \mathrm{X}$ & 0,89 & $* *$ \\
Heteranthera limosa & Pontederiacae & $\mathrm{Y}=28,43-3,52 \mathrm{X}$ & 0,94 & $* * *$ \\
\hline
\end{tabular}

Nota: Ecuaciones significativas a los niveles de $0,01 * *$ y $0,001 * * *$ respectivamente.

sativa (arroz rojo), Echinochloa colona y Cyperus spp., altas cantidades de rastrojos de arroz ( $9 \mathrm{t} / \mathrm{ha}$ ) equivalentes al doble de la cantidad estimada de rastrojos remanentes en campos arroceros de la región Pacífico Norte, no fueron suficientes para impedir completamente la emergencia de plántulas de estas especies por su efecto selectivo (Blum et al. 1997; Ilnicki y Enache 1992).

Al igual que el arroz y otros cultivos de gramíneas, casi todas las líneas de Oryza sativa (arroz rojo) no requieren irradiación luminosa para su germinación (Chung y Paek 2003). El comportamiento similar al arroz de Echinochloa colona a partir de las de 4,5 t de cobertura sugiere que también es una especie no fotoblástica o con una germinación insensible a la falta de luz. Según Fisher (2004) dejar rastrojos picados sobre el suelo e inundar es más efectivo que enterrarlos para el control de especies del mismo género Echinochloa, incluyendo biotipos resistentes a herbicidas.

De todas las especies, las más tolerantes a los distintos niveles de rastrojos ( 0 a $9 \mathrm{t} / \mathrm{ha}$ ) fueron Cyperus spp., Oryza sativa (arroz rojo) y Echinochloa colona, respectivamente. La mayor emergencia de plántulas de ciperáceas a los niveles más altos de rastrojos puede explicarse por la poca penetración de luz infraroja al suelo (Majek y Neary 1991) y por poseer estructuras apicales puntiagudas y resistentes que pueden penetrar con mayor facilidad a través de los rastrojos (Patterson 1996, 1997).

A diferencia de las especies de Cyperus evaluadas en este estudio, la germinación de Fimbristylis spp. requiere luz y es más sensitiva a la cobertura con rastrojos. La menor emergencia de Fimbristylis a niveles bajos de cobertura y la no emergencia a niveles altos puede deberse a la falta de luz. En estudios previos se ha demostrado que semillas de este género no germinan en la oscuridad aún cuando las semillas no están dormantes y son expuestas a temperaturas fluctuantes (Schütz 2000).

Las semillas de diferentes especies pueden tener distintos requerimientos de luz para su germinación (Pons 1992). La inhibición de la germinación de Ludwigia y Heteranthera limosa en la oscuridad producida por coberturas de rastrojos superiores a las 7,9 t/ha es consistente con lo que ocurre con especies que forman bancos de semillas persistentes (Grime et al. 1985). También el efecto físico de los rastrojos reduce la posibilidad de desarrollo de plántulas de malezas con estructuras frágiles y cantidades pequeñas de reservas en los diásporos (Pitelli y Durigan 2001). Adicionalmente, la liberación de aleloquímicos en los exudados de los rastrojos de arroz en descomposición puede suprimir el desarrollo de malezas. Se ha demostrado que estos aleloquímicos controlan Heteranthera limosa y otras malezas acuáticas (Dilday et al. 1991). En un estudio llevado a cabo en Arkansas, rastrojos de varias líneas de arroz controlaron más del $80 \%$ de Heteranthera limosa y Ammania coccinea (Lin et al. 1992).

Los efectos alelopáticos de los exudados de la descomposición de los rastrojos de arroz son de acción prolongada y están directamente relacionados con la cantidad de rastrojos (Almeida 1991). Recientemente, se ha confirmado que las raíces de arroz liberan cantidades de momilactone B (3,20-epoxy-3 $\alpha$-hydroxy9 $\alpha$-pimara-7,15-dien-19,6 $\alpha$-olide) suficientes para inhibir el desarrollo de malezas (Kato-Noguchi 2004). Es posible que esta sustancia en combinación con ácidos fenólicos liberados en los exudados de los rastrojos produzca efectos alelopáticos (Olofsdotter 2001). Los efectos alelopáticos de los rastrojos combinados con la 
falta de luz y otros efectos físícos inciden en la emergencia de las plántulas de malezas (Jones et al. 1999), y pueden ser la causa del control total de Ludwigia y Heteranthera limosa a partir de las 6,8 t/ha de rastrojos de arroz sobre el suelo.

De manera similar a las tendencias observadas en el presente estudio, Herrera (2002) determinó que cuando la cantidad de rastrojos de arroz variedad CR 5272 fue mayor a 5,6 t/ha, la densidad de malezas dicotiledóneas y poáceas fue sumamente baja durante el período crítico de interferencia con el cultivo. La poca presencia de malezas en estas condiciones pudo deberse a que los rastrojos redujeron drásticamente la cantidad de luz fotosintéticamente activa y el total que penetró en el suelo. En contraste con lo que ocurre con la incorporación de los rastrojos en SMC, dejarlos sobre el suelo en SDR permite reducir la diversidad, densidad y frecuencia de poblaciones de malezas, independientemente del estado inicial de la flora de malezas antes de la implementación de diferentes sistemas de labranza (Bilalis et al. 2003).

En concordancia con estudios realizados por Olofsdotter (2001), habría que validar mediante análisis de laboratorio si los resultados de esta investigación se deben en parte a propiedades alelopáticas de los exudados de los rastrojos de la variedad CR 1113. Si este fuera el caso, sería factible controlar efectivamente algunas malezas problemáticas asociadas al cultivo mediante los exudados de este tipo de rastrojos. Combinando el manejo de los rastrojos de arroz con otras alternativas para el control de malezas, se podrían poner en práctica programas integrales que permitan reducir el uso de herbicidas y alcanzar niveles superiores de efectividad (Herrera 2002; Williams et al. 1998). Para lograr un uso efectivo de herbicidas preemergentes aplicados en programas integrales de control de malezas en SDR, debe tomarse en cuenta que su intercepción y retención por rastrojos en la superficie del suelo puede limitar parcial o totalmente el contacto con semillas de las malezas y reducir su bioeficacia (Reddy et al. 1995).

\section{Emergencia del arroz bajo cobertura de rastrojos del mismo cultivo}

Los resultados del segundo experimento mostraron que las coberturas con rastrojos de arroz inferiores a 4,5 t/ha no afectaron significativamente los porcentajes de emergencia del arroz CR 1113 (Figura 2). Por encima de dicha cifra se observó una relación inversa $\left(\mathrm{R}^{2}=\right.$ 0,96) con un mínimo de $47 \%$ de emergencia de plántulas para la mayor cobertura con rastrojos ( $9 \mathrm{t} / \mathrm{ha})$. En general la germinación del arroz no es afectada por la

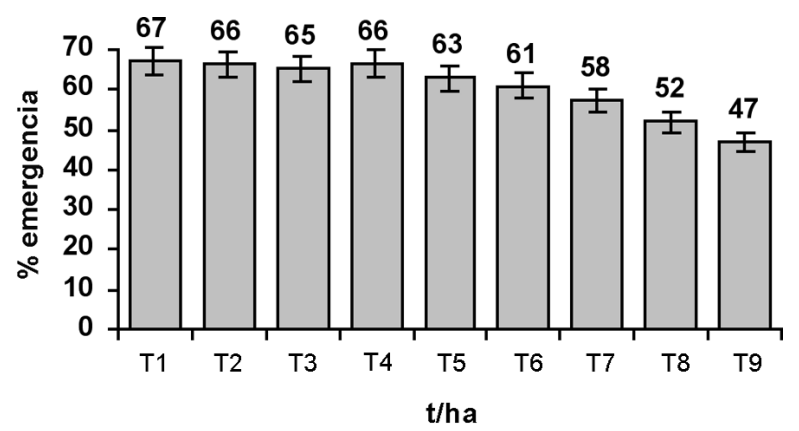

Figura 1. Emergencia de plántulas de arroz CR1113 (\%) según diferentes intensidades de cobertura con rastrojos del mismo cultivo (t/ha). Hacienda. El Pelón, Guanacaste, Costa Rica. 2003.

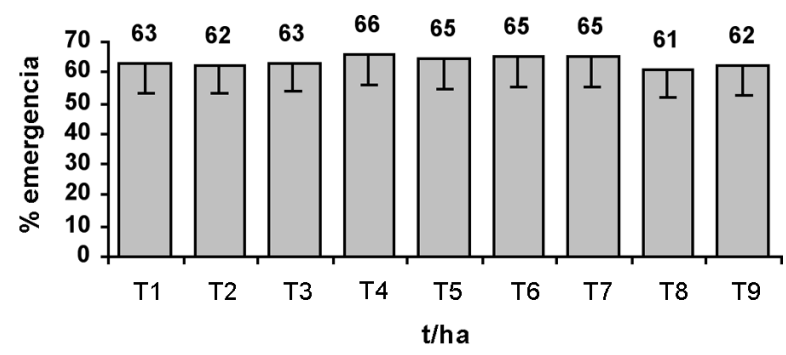

Figura 2. Emergencia de plántulas de arroz CR1113 (\%) según diferentes cantidades de cobertura con rastrojos de frijol negro Huasteco(t/ha). Hacienda. El Pelón, Guanacaste, Costa Rica. 2003.

oscuridad o la luz, y es poco común encontrar biotipos fotoblásticos con germinación favorecida por irradiación luminosa (Chung y Paek 2003).

Contrario a lo que ocurre con semillas fotoblásticas, la luz roja lejana que pasa a través de los rastrojos no evita el proceso de germinación del arroz. Aún bajo condiciones favorables las semillas fotoblásticas positivas permanecen dormantes hasta que son sacadas a la superficie. Estas semillas detectan que la luz roja lejana favorable para la fotosíntesis no es adecuada para el crecimiento de las plántulas y en consecuencia no se produce la germinación (Cone y Kendrick 1985). El arroz al igual que otras especies con semillas insensitivas a la luz, generalmente germina tanto en la oscuridad como bajo cualquier longitud de onda luminosa (Takaki 2001). Al igual que el arroz rojo, el arroz comercial rara vez requiere de luz para su germinación. Esta puede ser la principal causa de una emergencia superior al $45 \%$ en todos los niveles de cobertura ( 0 a $9 \mathrm{t} / \mathrm{ha}$ ) considerados en este estudio. La mayor y más uniforme emergencia del arroz en comparación con el arroz rojo, puede deberse a la menor dormancia primaria de las variedades comerciales (Cohn y Hughes 1981). 
Los factores ambientales como la radiación solar, la temperatura y el contenido de humedad del suelo influyen en forma combinada en la germinación de las semillas. Las fluctuaciones de temperatura varían según sea la radiación solar y el efecto de las mismas depende de la humedad presente en el suelo. Hasta ciertos niveles de cobertura, los rastrojos reducen la evaporación y conservan la humedad sin impedir que la radiación solar origine las fluctuaciones de temperatura necesarias para inducir la germinación del arroz. Cuando la cantidad de rastrojos es excesiva, la combinación resultante de estos factores no es favorable para que las semillas del cultivo germinen y en parte ésta puede ser la causa de la reducción en la germinación del arroz conforme la cantidad de rastrojos fue mayor.

La reducción en la germinación del arroz a altos niveles de cobertura con rastrojos de ese mismo cultivo podría además obedecer a problemas de autointoxicación, los cuales pueden presentarse particularmente en campos arroceros bajo monocultivo continuo. Aunque se ha indicado que extractos acuosos de residuos de arroz en descomposición reducen el crecimiento de arroz, y otras plántulas mono y dicotiledóneas (Chow 1998; Olofsdotter 2001), investigaciones desarrolladas por Herrera (2002) no indican evidencia de que los mismos causaran efectos alelopáticos en frijol. En otro estudio, Rimando et al. (2001) indican que faltan más estudios para explicar los efectos alelopáticos del arroz. Avances logrados recientemente por Kato-Noguchi (2004) sugieren que el momilactone B puede jugar un rol importante en la alelopatía del arroz, pero siguen sin comprenderse claramente el modo de acción y las interacciones entre los aleloquímicos de los cultivares.

\section{Emergencia del arroz bajo cobertura de rastrojos de frijol}

En el tercer experimento no se encontró evidencia de que las coberturas con rastrojos evaluadas afectaran la germinación del arroz. Por ser rastrojos frágiles con una favorable relación $\mathrm{C}: \mathrm{N}$, normalmente presentan una tasa de descomposición más rápida, y la cobertura de éstos sobre el suelo desaparece con mayor prontitud. Aunque esta clase de rastrojos no restringió la germinación del arroz, tampoco constituyó una cobertura duradera para mitigar procesos de erosión hídrica o eólica en condiciones de campo.

Estudios realizados por White et al. (1989) indican que las plantas leguminosas pueden producir compuestos alelopáticos. Sin embargo, otros autores reportan que los efectos de los residuos tienen un efecto selectivo (Blum et al. 1997) y la liberación de $\mathrm{N}$ fijado sim- bióticamente favorece el desarrollo de plántulas de cultivos y malezas (Teasdale 1996). Además de la contribución de nitrógeno, el cultivo de frijol en rotación con arroz puede reducir la incidencia de plagas y enfermedades y minimizar el desarrollo de resistencia en especies dañinas.

A pesar de que en este estudio no hubo evidencia de daños al cultivo, algunos investigadores (Kimber 1973; Mason-Sedun et al. 1986) indican que existen efectos negativos potenciales asociados a rotaciones de cultivo, en especial cuando hay grandes cantidades de residuos frescos en contacto con el cultivo de turno. Adicionalmente, algunos herbicidas tales como fluometuron e imazetapir usados en rotaciones con cultivos de leguminosas pueden persistir y ocasionar daños fitotóxicos al arroz plantado en años siguientes.

\section{CONCLUSIÓN}

Aunque los rastrojos de arroz al formar coberturas superiores a 4,5 t/ha limitaron los procesos de germinación y emergencia tanto de malezas comunes en el agroecosistema arrocero inundado como del propio cultivo; bajo las condiciones regulares de campo, cuando lo normal es que la densidad de estos residuos vegetales sea inferior al total producido del ciclo previo, la posibilidad de que ocurra tal efecto debe ser menor. En consecuencia, al adoptar el sistema SDR no hay evidencia de que la cobertura de rastrojos contribuya a disminuir la incidencia de malezas en el cultivo y es inconveniente disminuir la intensidad de los tratamientos para el control preemergente de malezas. Por la misma razón, tampoco será preciso ajustar la densidad de siembra del arroz con respecto a la que se aplica en sistemas de cultivo convencionales.

Cuando se adopta la SDR, el principal beneficio en el manejo de malezas resulta de no disturbar el terreno, agotándose el banco de semillas de malezas en la capa más superficial del suelo luego de varios ciclos. La reducción ocurre siempre y cuando se elimine con eficiencia las malezas que emerjan durante los primeros ciclos del cultivo con esta tecnología. Este aspecto tiene mucho mayor peso en el manejo de malezas, que la potencial inhibición por los rastrojos. Es recomendable evaluar posibles efectos de la acumulación de rastrojos de arroz, luego de varios ciclos de siembra de arroz en monocultivo, para comprobar si las tendencias son distintas a las determinadas con coberturas correspondientes a un solo ciclo previo y si se producen efectos en los rendimientos del cultivo. 


\section{LITERATURA CITADA}

ALMEIDA, F.S. 1991. Controle de plantas daninhas em plantio directo. IAPAR. Londrina, Brazil. Circular 67. 34 p.

BESNIER, R. 1989. Semillas: biología y tecnología. Mundi-prensa, Madrid. 637 p.

BILALIS, D.; SIDIRAS, N.; ECONOMOU, G.; VAKALI, C. 2003. Effect of different levels of wheat straw soil surface coverage on weed flora in Vicia faba crops. Journal of Agronomy and Crop Science 189 (4): 233-239.

BLUM, U.; KING, L.D.; GERIG, M.E.; LEHMAN, M.E.; WORSHAM, A.D. 1997. Effects of clover and small grain cover crops and tillage techniques on seedling emergence of some dicotyledonous weed species. Am. J. Altern. Agric. 4: 146-161.

BULL, L.1993. Residue and tillage systems for field crops. USDA Economic research service. Staff Report No. AGES 9310. Whasington, DC. 15 p.

CCT (Centro Científico Tropical, C.R.). 1993. Mapa ecológico de Costa Rica, según el sistema de clasificación de zonas de vida del mundo de L.R. Holdridge. Hoja Nicoya. Escala 1:200,000.

CHOW, C.H. 1998. Adaptative autointoxication in rice. In: Allelopathy in rice. IRRI, Filipinas 154 p.

CHUNG, N.J.; PAEK, N.C. 2003. Photoblastism and ecophysiology of seed germination in weedy rice. Agron. J. 95: 184-190.

COHN, M.A.; HUGHES, J.A., 1981. Seed dormancy in red rice (Oryza sativa): I. Effect of temperature on dry-after-ripening. Weed Sci. 29: 402-404.

CONE, J.W.; KENDRICK, M. 1985. Fluence-response curves and action spectra for promotion and inhibition of seed germination in wild type and long hypocotyl mutants of Arabidopsis thaliana L. Planta 163: 43-54.

CORDERO, V. A. 1993. Fertilización y nutrición mineral del arroz. EUCR, San José. C.R. 100 p.

CORREIA, N.M.; DURIGAN, J.C. 2004. Emergência de plantas daninhas em solo coberto com palha de canade-açúcar. Planta Daninha 22(1): 11-17.

DILDAY, R.H.; NASTASI, P.; LIN, J.; SMITH, R.J. 1991. Allelopathic activity of rice (Oryza sativa L.) against ducksalad [Heteranthera limosa (Sw.) Willd.]. In: Proc.
Symposium sustainable agriculture for the great plains. Hanson J.D., Shaffer M.J., Ball D.A., Cole C.V. (eds.). ARS-89. U.S. Department of Agriculture. Agricultural Research Service, Washington, D.C., U.S.A., p. 193201.

FADEL, J.G.; MACKILL, D.J. 1994. Characterization of rice straw. California Rice Research Board. Consultado 14 oct. 2004. Disponible en: http://www.syix.com/rrb/94rpt/RiceStraw.htm.

FAULKNER, E.H. 1943. Plowman's folly. Grosset \& Dunlap Pub. New York. 117 p.

FISHER, A. 2004. Weed control in rice-01. California Rice Research Board. Consultado 14 oct. 2004. Disponilble en: http://www.syix.com/rrb/01rpt/Weed. htm.

FUKUOKA M. 1978. The one straw revolution. Rodale Press, Inc. New York 181 p.

GRIME, J.P.; SHACKLOCK, J.M.; BAND, S.R. 1985. Nuclear DNA contents, shoot phenology and species co-existence in a limestone grassland community. New Phytologist 100: 435 - 445 .

HERRERA, F. 2002. Manejo de rastrojos, las malezas y el arroz voluntario en un sistema de rotación frijol-arroz. Tesis doctoral, San José, Costa Rica. Universidad de Costa Rica. 182 p.

IGN (Instituto Geográfico Nacional, C.R.). 1987. Mapa básico de Costa Rica,. Hoja Tempisque 3147-III, Escala $1: 50,000$.

ILNICKI, R.D.; ENACHE, A. J. 1992. Subterranean clover living mulch: an alternative method of weed control. Agric. Ecosyst. Technol. 40: 249-264.

JASA P., SHELTON D., SIEMENS, J. 2000. Tillage system selection and equipment considerations. In: Conservation tillage systems and management. MWPS-45, 2a ed. p. 186-190.

JOHNSTON, T.H.; MILLER, M.D. 1973. Culture. in:Rice in the United States: varieties and production. USDAARS Handbook 289. U.S. Department of Agriculture, Washington D.C. p. 88-134.

JONES, E.; JESSOP, R.S.; SINDEL, B.M.; HOULT, A. 1999. Utilising crop residues to control weeds. In: Bishop A., Boersma M.; Barnes C.D. (ed.). Proceedings of the 12th Australian Weeds Conference. Tasmanian Weeds Society, Devonport. p. 373-376. 
KATO-NOGUCHI H. 2004. Allelopathic substance in rice root exudates: Rediscovery of momilactone B as an allelochemical. Journal of Plant Physiology 161(3): 271-276.

KIMBER, R.W.L. 1973. Phytotoxicity from plant residues. II. The effect of time of rotting of straw from some grasses and legumes on the growth of wheat seedlings. Plant and Soil 38: 347-61.

LIN, J.; SMITH, R.J.; DILDAY, R.H. 1992. Allelopathic activity of rice germplasm on weeds. Proc. Southern Weed Science Society 45: 99.

MAJEK, B.A.; NEARY, P.E. 1991. Selective wavelength transmitting mulch for yellow nutsedge control. In: Proceedings 1991 Brighton Crop Protection Conference- Weeds. Brighton, UK. p. 263-268.

MASON-SEDUN, W.; JESSOP, R.S.; LOVETTm J.V. 1986. Differential phytotoxicity among species and cultivars of the genus Brassica to wheat. Plant and Soil 93: 3-16.

OLOFSDOTTER, M. 2001. Rice-A step toward use of allelopathy. Agron. J. 93: 3-8.

OLOFSDOTTER, M.; REBULANAN, M.; MADRID, A.; DALI, W.; NAVAREZ, D.; OLK, D.C. 2001. Why phenolic acids are unlikey allelochemicals in rice. Journal of Chemical Ecology 28 (1) 229-242.

PATTERSON, D.T. 1996. Suppression of purple nutsedge with plastic mulch. In: Annual international conference on methyl bromide alternatives and emissions reductions. Orlando, Florida. p. 11-1 - 11-2.

PATTERSON, D.T. 1997. Factors affecting the suppression of purple nutsedge by translucent plastic film mulch. In: Annual International Conference on Methyl Bromide Alternatives and Emissions Reductions. San Diego, California, USA. p. 18-1 - 18-2.

PENFOLD C. 2003. Weed suppression with mulches. Viticare News. Volume 4, Issue 11. 9 p.

PENNY, G.M.; NEAL, J.C. 2003. Light, temperature, seed burial, and mulch effects on mulberry weed (Fatoua villosa) seed germination. Weed Technology 17:213-218.

PITELLI, R.A.; DURIGAN, J.C. 2001. Ecologia das plantas daninhas no sistema de plantio directo. In: Rosello R.D. Siembra directa en el con sur. Montevideo: PROCISUR. p. 203-210.
PONS, T.J. 1992 Seed responses to light. Seeds. The ecology of regeneration in plant communities. Ed. Fenner M. CAB International, New York. p. 259-284.

QUICK, G.R. 2003. Rice harvesting. In: Rice origin, history, technology and production. Smith C.W and Dilday R.H. Eds. John Willey \& Sons Inc, p. 491-543.

REDDY, K.N. 2001. Effects of cereal and legume crop residues on weeds, yield, and net return in soybean (Glycine $\max$ ). Weed Technology 15: 660-668.

REDDY, K.N.; LCKE, M.A.; WAGNER, S.C.; ZABLOTOWICZ, R.M.; GASTON, L.A.; SMEDA, R.J. 1995. Chlorimuron ethyl sorption and desorption kinetics in soils and herbicide-desiccated cover crop residues. J. Agric. Food Chem. 43:2752-2757.

RIMANDO, A.M.; OLOFSDOTTER M.; DUKE, S.O. 2001. Searching for rice allelochemicals. Agron. J. 93:16-20.

SALISBURY, F.B.; ROSS C.W. 1992. Fisiología vegetal. Grupo Editorial Iberoamericana, México 759 p.

SCHÜTZ, W. 2000. Ecology of seed dormancy and germination in sedges (Carex). Perspectives in plant ecology, Evolution and Systematics. 3 (1): 67-89.

SHELTON, D.; JASA, P.; BROWN, L.; HIRSCHI, M. 2000a. Water erosion. In: Conservation tillage systems and management. MWPS-45, $2^{\mathrm{a}}$ ed. $19 \mathrm{p}$.

SHELTON, D.; SMITH, J.; JASA, P. 2000b. Estimating residue cover. In: Conservation tillage systems and management. MWPS-45, $2^{\text {a }}$ ed. p. 29-34.

SHRESTHA, A.; KNEZEVIC, S.Z.; ROY, R.C.; BALLCOELHO, B.R.; SWANTON, C.J. 2002. Effect of tillage, cover crop and crop rotation on the composition of weed flora in sandy soil. Weed Research 42(1): 76-87.

SIMMONS, F.W. 2002. Illinois Agronomy Handbook. 23 ed. USA, $318 \mathrm{p}$.

SMITH, J.; HOFFMAN, V.; TAYLOR, R. 2000. Residue Management at harvest. In: Conservation tillage systems and management. MWPS-45, $2^{\text {a }}$ ed. p. 29-34.

TAKAKI, M. 2001. New proposal of classification of seeds based on forms of phytochrome instead of photoblastism. Rev. Bras. Fisiol. Veg. 13(1):104-108.

TEASDALE, J.R. 1996. Contribution of cover crops to weed management in sutainable agricultural systems. J. Prod. Agric. 9:475-479. 
TEMPLE, S.R.; SOMASCO, O.A.; KIRK, M.; FRIEDMAN D. 1994. Conventional, low-input and organic farming systems compared. California Agriculture 48(5): 15-20.

WEBER, S.; STUBNER, S.; CONRAD, R. 2001. Bacterial populations colonizing and degrading rice straw in anoxic paddy soil. Applied and Environmental Microbiology 67(3): 1318-1327.

WHITE, R.H.; WORSHAM, A.D.; BLUM, U. 1989. Allelopathic potential of legume debris and aqueous extracts. Weed Sci. 37: 674-679.
WILLIAMS, M.M.; MORTENSEN, D.A.; DORAN J.W. 1998. Assessment of weed and crop fitness in cover residues for integrated weed management. Weed Sci. 46: 595-603.

YANG H, S.; KIM D, J. 2003. Rice straw-wood particle composite for sound absorbing wooden construction materials. Bioresource Technology 86(2) 117-121. 